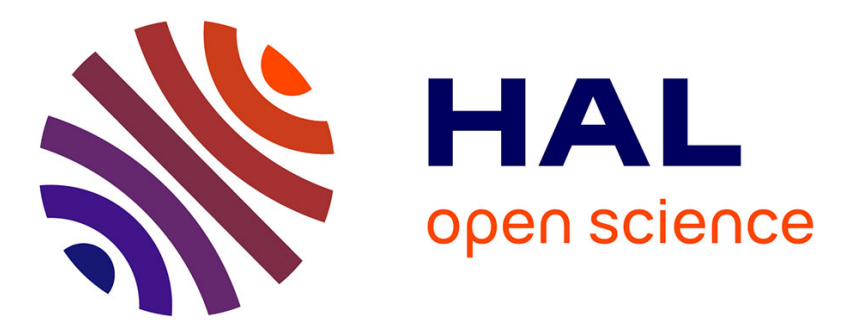

\title{
Strain heterogeneities and local anisotropy in crimped glass wool
}

Sandra Bergonnier, François Hild, Jean-Baptiste Rieunier, Stéphane G. Roux

\section{To cite this version:}

Sandra Bergonnier, François Hild, Jean-Baptiste Rieunier, Stéphane G. Roux. Strain heterogeneities and local anisotropy in crimped glass wool. Journal of Materials Science, 2005, 40, pp.5949-5954. $10.1007 / \mathrm{s} 10853-005-5068-8$. hal-00017630

\section{HAL Id: hal-00017630 https://hal.science/hal-00017630}

Submitted on 18 Sep 2008

HAL is a multi-disciplinary open access archive for the deposit and dissemination of scientific research documents, whether they are published or not. The documents may come from teaching and research institutions in France or abroad, or from public or private research centers.
L'archive ouverte pluridisciplinaire HAL, est destinée au dépôt et à la diffusion de documents scientifiques de niveau recherche, publiés ou non, émanant des établissements d'enseignement et de recherche français ou étrangers, des laboratoires publics ou privés. 


\title{
Strain heterogeneities and local anisotropy in crimped glass wool
}

\author{
Sandra Bergonnier ${ }^{(1,2)}$, François Hild ${ }^{(1)}$, Jean-Baptiste Rieunier ${ }^{(3)}$, \\ Stéphane Roux ${ }^{(2)}$ \\ (1) LMT-Cachan, ENS de Cachan/CNRS-UMR 8535/Université Paris 6 \\ 61 Avenue du Président Wilson, F-94235 Cachan Cedex, France. \\ (2) Surface du Verre et Interfaces, Unité Mixte de Recherche CNRS/Saint-Gobain 125 \\ 39 Quai Lucien Lefranc, 93303 Aubervilliers Cedex, France. \\ (3) Saint-Gobain Isover - CRIR, BP 10019, 60291 Rantigny, France.
}

January 10, 2005

\begin{abstract}
A Digital Image Correlation method is used to study a compression test carried out on crimped glass wool samples to determine the displacement field, which reveals strain heterogeneities and further localization. The structure of the wool is locally strongly anisotropic, even though the preferential orientation varies significantly in space. This structure is analyzed using an ad hoc orientation detection tool. It is shown that the strain amplitude can be correlated with texture gradients for this type of material.
\end{abstract}

Keywords: compression test, digital image correlation, orientation analysis, strain localization

\section{Introduction}

Mineral wool is a naturally textured material, well-known for its thermal and acoustic insulation properties. For some particular applications, mechanical performances are also required such as compression and tearing strengths. To evaluate the mechanical performances of the material and to quantify the effect of process parameters, mechanical tests are carried out.

To analyze tests performed on such a soft material, a contact-less method is needed. Moreover the method has to be able to deal with high strain levels. Digital Image Correlation (DIC) is particularly suited to that type of analysis. This technique is easy to use and has proven to be an efficient tool whose resolution can be extended much below the pixel accuracy (i.e., $10^{-2}$ pixel or below in suited cases). To reach high strain levels as for mineral wool tests with a very good resolution, a multiscale approach of DIC (Hild et al., 2002) can be used. It allows for large displacements between images with both a very good robustness and resolution.

Mineral wool being a highly textured material because of its natural fibrous character, it is interesting to look for a relationship between local anisotropy and mechanical strain. A tool that enables one to estimate the anisotropy of images or zones of images and the corresponding orientation field is needed. While DIC provides a strain field from mechanical tests, a texture analysis gives access to the initial orientation field of the tested sample. 
The purpose of the study is to present an analysis of a compression test using both DIC and texture analysis methods. Section 2 introduces the crimping process. Section 3 deals with the DIC method. The multiscale approach and the choice of the correlation parameters are discussed. Section 4 presents the texture analysis method and its results on, first, a test case, and then on the crimped glass wool sample. Last, the mechanical test carried out on the same crimped glass wool sample is analyzed in Section 5. Compression test is performed up to $-20 \%$ global strain and the relationship between the strain field and the initial texture is investigated.

\section{Crimped glass wool}

Glass wool is a cellular solid made of glass fibers whose diameter is in the range 1-5 $\mu \mathrm{m}$, and millimetric or centimetric length. Those fibers are entangled to form a very loose mat (a few $\mathrm{kg} / \mathrm{m}^{3}$ ). A binder sprayed on the fibers, and cured in an oven, freezes the arrangement of the fibers and provides some elasticity to the product. As such this gives a very good insulating material, with however little mechanical performances. For applications that are more demanding on the mechanical side, the fiber mat is processed before the curing stage to produce a structure endowing the material with a stronger mechanical resistance (to compression, tearing, or shear). This step is called "crimping". It consists in breaking the naturally layered structure of the mat, through an on-line transverse and axial compression. The denser layers are buckled in the axial compression, and produce a more isotropic fiber orientation (Fig. 1), and in particular a larger population of vertical fibers, whose mechanical stiffness and strength are much larger than for the initially layered material. This underlines the key role of the product texture for its mechanical behavior, and hence a proper characterization of both the local texture, and the local strain (and their interdependence), is required to optimize the product. This motivates the study detailed in Section 5.

\section{Displacement measurement by digital image correlation}

DIC is based on the use of an image matching algorithm (i.e., correlation product), which can be performed either in the physical space (Sutton et al., 1983; Chu et al., 1985) or in Fourier space (Chen et al., 1993; Berthaud et al., 1996). In the present case, Fourier computations are used. The in-plane displacement field is evaluated with the cross-correlation of an interrogation window in the picture of the deformed surface with respect to a reference one. To determine the displacement field of one image of the deformed surface with respect to a reference image, one considers a sub-image that will be 


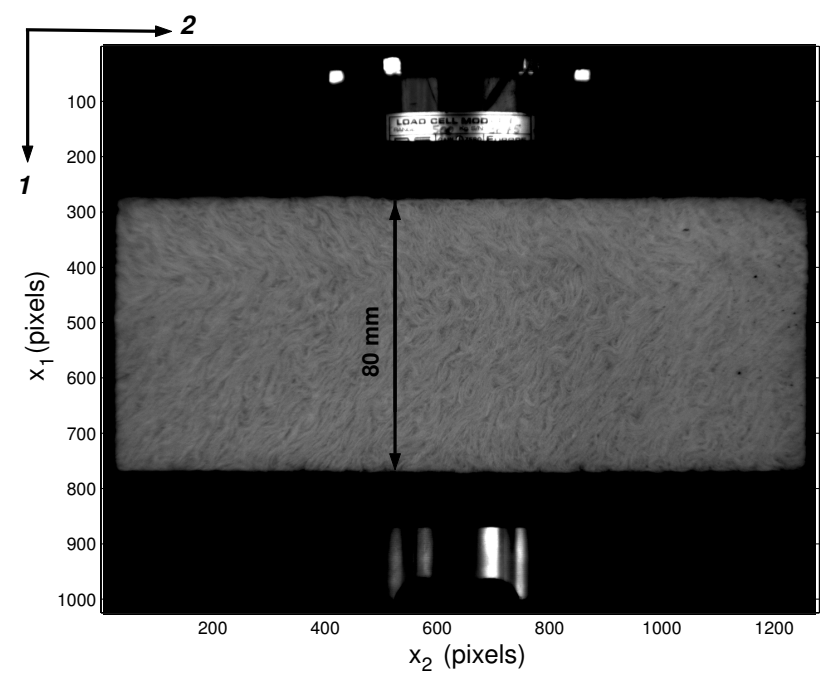

Figure 1. Lateral view of a crimped glass wool product (1 pixel $\Leftrightarrow 0.15 \mathrm{~mm})$.

referred to as a Zone Of Interest (ZOI). The aim of the correlation method is to match the ZOIs in the two images (Fig. 2). The displacement of a ZOI
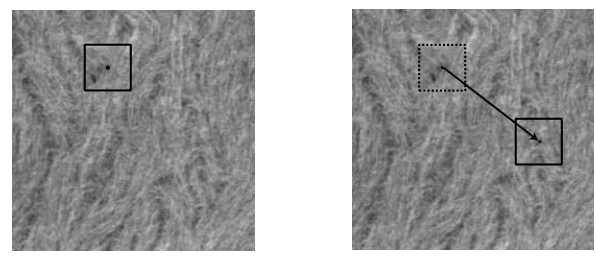

Figure 2. Zone of Interest (ZOI) in reference (left) and "deformed" (right) images. Associated displacement vector.

with respect to the other one is a two-dimensional shift of an intensity signal digitized by a CCD camera. In practice, two images are considered. The first one, referred to as 'reference image' and the second one, called 'deformed image'. The reference image, schematically shown in Fig. 3, is meshed by ZOIs that form the region of interest (ROI). The sub-ROI of size $2^{P} \times 2^{Q}$, is extracted from the ROI in the reference image with the largest values of $P$ and $Q$.

First a standard approach can be used (Périé et al., 2002), which is wellsuited for small strain perturbations. But if displacements are too large, the information in the ZOI of the reference image will be partially lost in the ZOI of the deformed image, i.e., there is not enough information to determine a reliable correction. To use the method in the domain of large strains, as for the case of mineral wool, a large ZOI size should be used, whose main drawback is to average displacements over a larger surface. Yet the aim of a correla- 


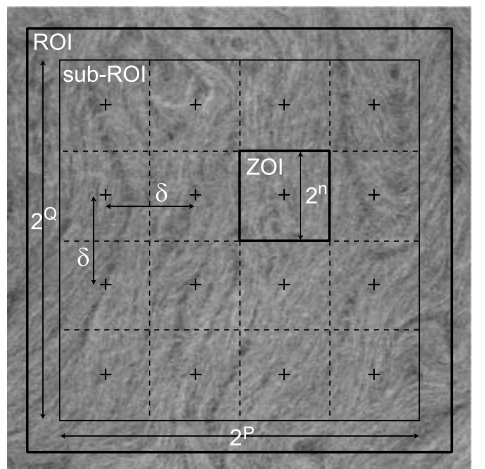

Figure 3. Schematic of the reference image with the correlation parameters (ZOI area: $2^{n} \times 2^{n}$ pixels and shift $\delta$ ).

tion calculation is to determine displacement fields at the scale of texture heterogeneities. The maximum measurable displacement for the algorithm to converge to the proper solution has then to be increased. This is achieved by using a multiscale algorithm.

\subsection{Multiscale Algorithm}

The aim of the multiscale algorithm is to improve the spatial resolution (i.e., to decrease the ZOI size), yet allowing for a large displacement between two consecutive images. The previous approach is recursively applied on images at different scales. First the average strains are deduced from an analysis with images where finer details have been erased (i.e., super-pictures, as shown in Fig. 4). To make the evaluation more robust, the interpolated displacements are applied to the analyzed ZOI and re-evaluated until the maximum difference between two iterations is smaller than a chosen value. Details can then be restored in the image (i.e., resolving finer scales) and the displacement interpolation can be used as a first evaluation of the field at the new scale. This procedure is iterated until the scale corresponds to the initial ROI (with all its details). During the last step, the displacement is no longer interpolated and fluctuations, whose amplitudes are greater than the ones that can be reached with the standard approach, can be analyzed.

Figure 4 shows the construction of images at different scales. Scale no. 0 corresponds to the considered image and scale no. 1 to the region of interest. Scale no. 2, extracted from the ROI, is determined by the largest values for $P$ and $Q$ so that the corresponding size of the sub-ROI is $2^{P} \times 2^{Q}$ (Fig. 3 ). From scale no. 3 on, each transition is characterized by the definition of super-pixels. The latter are defined recursively from one scale to the next by averaging the gray levels of $2 \times 2$ neighboring pixels. This procedure is carried out until the minimum size of the sub-image is equal to 128 pixels. 


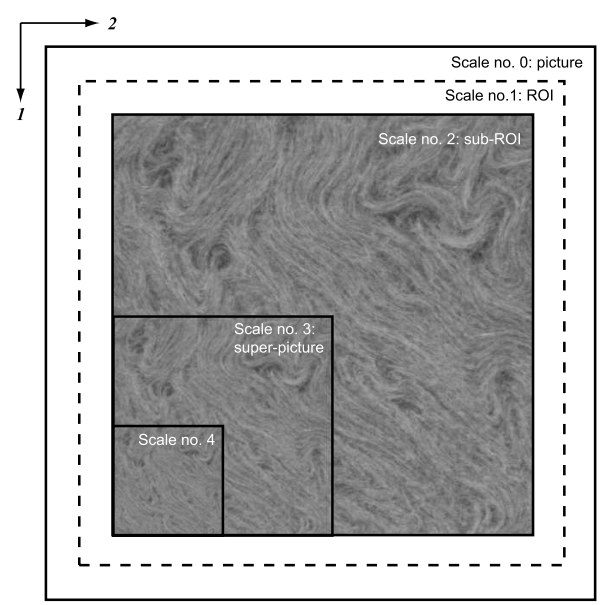

Figure 4. Different scales used in the multi-scale approach for a glass wool sample.

The analysis starts at, say, scale no. 4 and ends at scale no. 1 . This allows one to obtain a maximum measurable strain four times larger than the standard approach even if the maximum measurable displacement is still limited (Hild et al., 2002), as in the standard approach, only to the coarser scale (here scale no. 4). On artificially deformed images, strains of the order of $\pm 30 \%$ can be measured (Hild et al., 2002; Chevalier and Marco, 2002) between two pictures. When a sequence of more than 2 images is analyzed and when the reference image is updated (i.e., it corresponds to the deformed image of the previous step), there is no real limitation, apart from the fact that errors are cumulated.

\subsection{CHOICE OF THE CORRELATION PARAMETERS}

The aim of this part is to estimate the uncertainty of the correlation algorithm used herein. The displacement and strain uncertainties are estimated when the correlation parameters are modified. To have independent estimates of the displacements in the following analysis, the shift $\delta$ is equal to the ZOI size (i.e., $\delta=2^{n}$ pixels).

First, the displacement uncertainty is estimated. In the present case, a constant displacement of 0.5 pixel is applied artificially by using the shift/modulation property of Fourier transforms (Périé et al., 2002). The average difference gives an evaluation of the error, and the standard deviation that of the corresponding uncertainty. These properties are associated to the analyzed texture (Fig. 1). It can be shown that the displacement uncertainty is half that reached when the prescribed displacement is equal to 0.5 pixel (Bergonnier et al., 2004). Figure 5 shows the displacement uncertainty for different ZOI sizes. The larger the ZOI, the smaller the error. However, if the ZOI size 
increases, the spatial uncertainty is degraded, i.e., less independent measurement points are obtained.

Second, the strain uncertainty is determined by using the same picture. From the displacement field, in-plane strains are evaluated by using a classical finite differences scheme so that the spatial uncertainty (i.e., the gauge length) is equal to $2 \times \delta$, i.e., $2^{n+1}$ pixels. In all cases, the mean strain is less than $7 \times 10^{-5}$. The strain uncertainty is more sensitive to $\delta$ (here equal to the ZOI size) than the displacement uncertainty to the ZOI size (Fig. 5).

A good compromise for the present study is given by a ZOI size of 16 pixels (i.e., $n=4$ ) for which a displacement uncertainty of the order of 0.03 pixel is achieved. In terms of strains, the corresponding uncertainty is $3 \times 10^{-3}$ with a spatial uncertainty of 32 pixels. It should be remembered that all the results discussed so far were obtained with the same reference picture. When large strains or severe texture variations occur (Hild et al., 2002), an updating procedure is preferred. Consequently the uncertainty gradually degrades as the number of analyzed pictures increases.

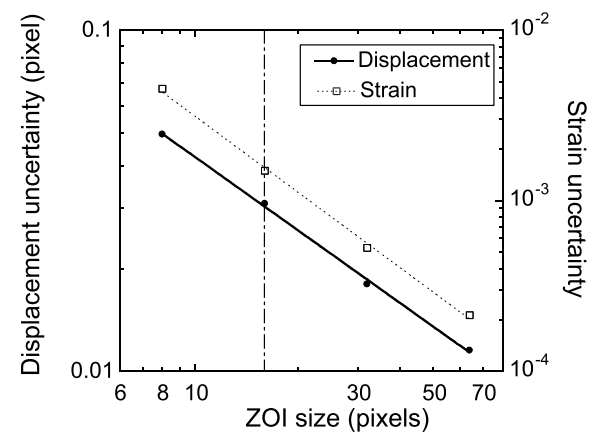

Figure 5. Displacement and strain uncertainties for different correlation parameters (ZOI size $=2^{n}$ pixels, shift $\delta=2^{n}$ pixels).

\section{Local anisotropy analysis}

Detecting the anisotropy of a domain, contained in images, involves an evaluation of the change of gray levels in different directions, the direction where the variation is the least being the principal axis of anisotropy. Since changes in gray levels are to be considered, most methods (Rao, 1990; Germain et al., 2003) are based on the calculation of gradient fields and implicitly assume a smoothness of the image. The method that is presented here does not require any smoothness assumption of the image. This may be an important improvement for images where the anisotropy is provided by very high frequency modes (i.e., fine texture). 
Let $f(x, y)$ represent a gray-scale image. The variation of gray levels suggests the use of an $L_{2}$-norm of the finite difference along the $e$ direction

$$
\left\|D_{\boldsymbol{e}} f\right\|=\left[\sum_{(x, y)}\left(f\left(x+e_{x}, y+e_{y}\right)-f(x, y)\right)^{2}\right]^{1 / 2}
$$

where $e_{x}, e_{y}$ are the components of the vector $e$. This $L_{2}$-norm can easily be computed for any direction in Fourier space. The total power is preserved in the Fourier transform, and thus

$$
\left\|D_{\boldsymbol{e}} f\right\|^{2}=\sum_{\mathbf{k}}(\mathbf{k} \cdot \mathbf{e})^{2}|\hat{f}(\mathbf{k})|^{2}
$$

Therefore, introducing the anisotropy tensor $\mathbf{T}$ defined as

$$
\boldsymbol{T}=\sum_{\mathbf{k}}(\mathbf{k} \otimes \mathbf{k})|\hat{f}(\mathbf{k})|^{2}
$$

one observes that

$$
\left\|D_{e} f\right\|^{2}=\mathbf{T}:(\mathbf{e} \otimes \mathbf{e})
$$

where ' $:$ ' is the tensorial contraction with respect to two indices. The anisotropy axis appears as the minor eigendirection of the anisotropy tensor $\mathbf{T}$. The anisotropy amplitude can be estimated conveniently through $\operatorname{dev}(\mathbf{T}) / \operatorname{tr}(\mathbf{T})$, where ' $\operatorname{tr}$ ' designates the trace, and 'dev' the norm of the deviator $\mathbf{T}-\operatorname{tr}(\mathbf{T}) \mathbf{I} / 2$.

\subsection{Application to A test image}

The method presented above is tested on concentric circles that represent an absolute reference because the true orientation is known at each position in the image. The image size is $512 \times 512$ pixels, and the size of the zones of interest $16 \times 16$ pixels. These zones are centered on a regular square grid and the distance between the center of two consecutive zones is 16 pixels as for the DIC analysis (Fig. 3).

In Fig. 6(a), where only one quarter of the image is shown, a first visual inspection gives a rough evaluation of the adequation between real orientations and estimates. Figure 6(b) shows the differences between the determined and true orientations as a function of the angle. The $0^{\circ}$ angle corresponds to a horizontal orientation. The RMS error or resolution is $0.63^{\circ}$.

\subsection{APPLiCATION TO CRIMPED GLASS WOOL}

The method is then applied to the determination of the texture orientation of a crimped glass wool sample. A special strategy has been used to limit the influence of edge effects (Bergonnier et al., 2005). For such an image (Fig. 1), 

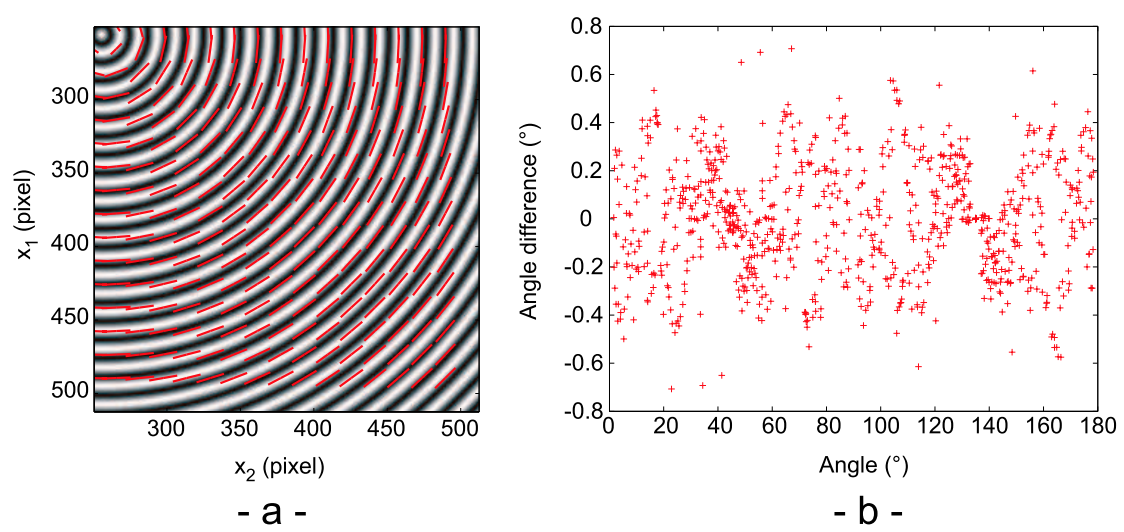

Figure 6. (a) Results of the anisotropy analysis on a test image, (b) Differences between calculated and true orientations.

it is to be noticed that there is a very fine texture, with a very difficult partition between noise and information carrying signal. Moreover, if anisotropy is clearly marked, the coherence length is rather short, and the pattern appears as circumvoluted. This is thus a very severe test of the proposed method.

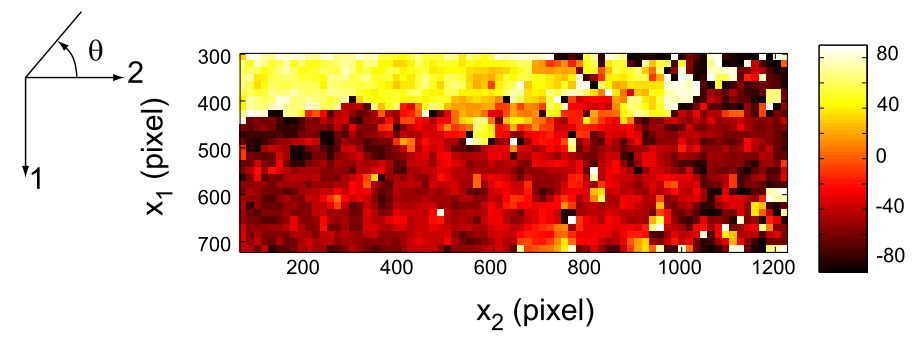

Figure 7. Texture orientation (in degrees) of the crimped glass wool sample before the mechanical test.

In Fig. 7, the texture orientation corresponding to the crimped glass wool sample (Fig. 1) is presented. A discontinuity of the orientation between the upper and the lower parts of the sample can be observed, with a $50^{\circ}$ orientation for the upper part and $\mathrm{a}-50^{\circ}$ orientation for the lower part. The fact that the texture is very fine can also be observed in Fig. 7 where the variations in the orientation field can be important from one ZOI to its neighbors. If the local texture of the sample has to be linked with the mechanical response of the sample to a compression test, the DIC method will enable one to compare the strain field and the initial orientation field by using the same calculation points. 


\section{Experiments: Uniaxial compression test on crimped glass wool}

The compression test reported herein is displacement-controlled (or equivalently, the global strain $\bar{\epsilon}$ is controlled; it is defined as the ratio of the displacement between the two platens divided by the initial height of the sample). A uniaxial compression (in the 1-direction) up to $-20 \%$ global strain is applied on parallelepipedic samples, whose dimensions are $200 \times 200 \times 80 \mathrm{~mm}^{3}$, $80 \mathrm{~mm}$ being the height of the sample (Fig. 1). For the test, images of the sample illuminated with direct light have been taken for different global strain levels (with $-1 \%$ increments in compression).

The result of the test is shown in Fig. 8. The "plastic" regime suggests the existence of heterogeneous strains. This implies to study the strain field of the material with a good spatial resolution. In Fig. 9, initial texture orientation and nominal strain fields are shown. For the latter, the analyzed kinematic quantity is evaluated for a reference given prior to the beginning of the experiment. The ZOIs used for the calculation correspond to the ZOIs used for the local anisotropy analysis. The calculation, performed with ZOIs of size $16 \times 16$ pixels with a 16 -pixel shift $\delta$, corresponds to the component $\epsilon_{11}$ for different global strain levels with an adaptive scale, namely, $-5 \%$ for Fig. 9(b), $-7 \%$ for Fig. 9(c), $-10 \%$ for Fig. 9(d) and $-15 \%$ for Fig. 9(e), respectively. In Fig. 8, the corresponding points are located in the elastic domain, at the end of the elastic domain, at the beginning of the "plastic" domain and where the "plastic" domain is well-defined. For the calculation of the strain fields the same filtering as for the anisotropy analysis has been applied. Within the elastic domain (Fig. 9(b-c)) a first strain localization zone

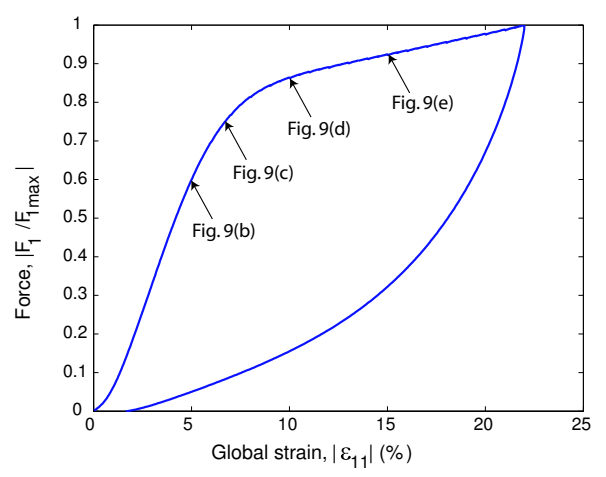

Figure 8. Dimensionless load vs. global strain for a compression test on crimped glass wool.

can be identified that seems to traverse the sample. For a $-10 \%$ global strain level (Fig. 9(d)), strain heterogeneities can well be observed at the surface of the material and a well-marked localized zone appears that correspond to 


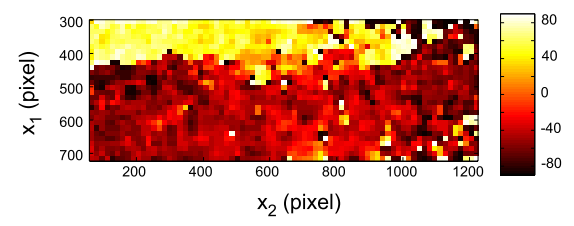

a. Initial texture orientation (in degrees)

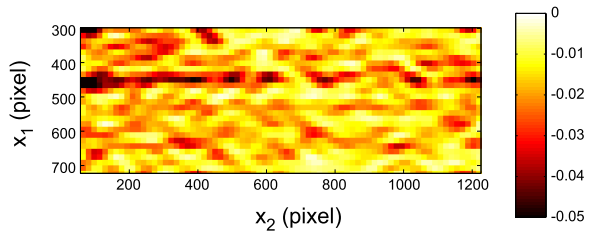

b. Cumulative field: $\bar{\epsilon}_{11}=-5 \%$

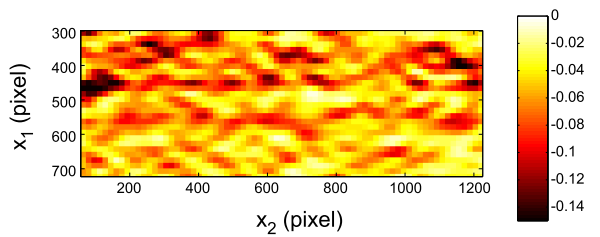

d. Cumulative field: $\bar{\epsilon}_{11}=-10 \%$

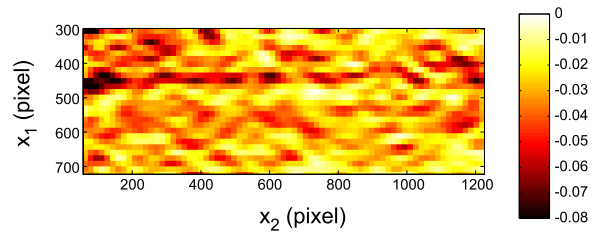

c. Cumulative field: $\bar{\epsilon}_{11}=-7 \%$

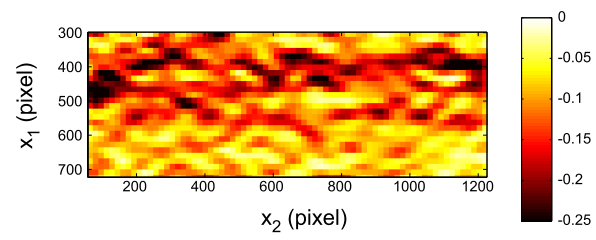

e. Cumulative field: $\bar{\epsilon}_{11}=-15 \%$

Figure 9. Initial texture orientation and cumulative $\epsilon_{11}$-field for different levels of the global strain. Correlation parameters: size of ZOI $=16 \times 16$ pixels, $\delta=16$ pixels. Scale: 1 pixel $\Leftrightarrow$ $0.15 \mathrm{~mm}$.

the first zone identified in Fig. 9(b-c). For $-15 \%$ global strain level, strain fluctuations appear around the previously observed localized zone.

The strain localization zone corresponds to a band that traverses the surface of the sample. If the orientation map (Fig. 9(a)) is compared to the strain fields, one can observe that the discontinuity in the orientation field is located where strain localization band occurs. The well-marked discontinuity zone on the left part of the material corresponds to where the first strain localization zone can be observed at early stages within the elastic domain (Fig. 9(b-c)).

\section{Conclusion}

First, a multiscale approach of the displacement and strain fields identification has been presented. The algorithm increases both the robustness and the maximum measurable displacement and strain between two consecutive images. This technique is well-suited to analyzing compression tests carried out on crimped glass wool samples.

Second, an image analysis tool has been introduced to determine the local anisotropy of an image. This approach gives access to the orientation field 
of an image. Validated on an artificial case, it was then applied on a crimped glass wool sample picture.

The results of a compression test on a crimped glass wool sample are studied. The DIC technique enables one to identify strain heterogeneity zones, and then strain localization bands. If both methods, DIC and local anisotropy analysis are coupled, the discontinuity zone in orientation can be linked to the localization bands in the strain field.

The correlation technique applied to crimped glass wool shows the change of macrostructure during the test. A further development is to characterize the change in the local orientation by the presented method and to relate it to strain localization.

\section{Acknowledgements}

This work has been supported by Isover Saint-Gobain and by CNRS in a project entitled "Analyses multi-échelles de champs de déformation par traitement d'image : vers l'identification de champs de propriétés mécaniques." The authors acknowledge useful discussions with M. Baudequin, O. Gaume and C. Machelart.

\section{References}

Bergonnier, S., Hild, F. and Roux, S.: Digital image correlation used for mechanical tests on crimped glass wool samples. J. Strain Analysis, accepted, 2004.

Bergonnier, S., Hild, F. and Roux, S.: Submitted for publication, 2005.

Berthaud, Y., Scholz, J. and Thesing, J.: Méthodes optiques et acoustiques de mesures des caractéristiques mécaniques. Proceedings Colloque national MECAMAT 'Mécanismes et mécanique des grandes déformations', 77-80, 1996.

Chen, D.J., Chiang, F.P., Tan, Y.S. and Don, H.S.: Digital Speckle-Displacement Measurement Using a Complex Spectrum Method. Appl. Opt., 32: 1839-1849, 1993.

Chevalier, L. and Marco, Y.: Tools for Multiaxial Validation of Behavior Laws Chosen for Modeling Hyper-Elasticity of Rubber-Like Materials. Polym. Eng. Sci., 42(2): 280-298, 2002.

Chu, T.C., Ranson, W.F., Sutton, M.A. and Petters, W.H.: Applications of Digital-ImageCorrelation Techniques to Experimental Mechanics. Exp. Mech., 3(25): 232-244, 1985.

Germain, C., Da Costa, J.P., Lavialle, O. and Baylou, P.: Multiscale estimation of vector field anisotropy application to texture characterization. Signal Processing, 83(7): 1497-1503, 2003.

Hild, F., Raka, B., Baudequin, M., Roux, S. and Cantelaube, F.: Multi-Scale Displacement Field Measurements of Compressed Mineral Wool Samples by Digital Image Correlation. Appl. Optics, 41(32): 6815-6828, 2002.

Périé, J.N., Calloch, S., Cluzel, C. and Hild, F.: Analysis of a Multiaxial Test on a C/C Composite by Using Digital Image Correlation and a Damage Model. Exp. Mech., 42(3): 318-328, 2002.

Rao, A.: A Taxonomy for Texture Description and Identification. Springer-Verlag, 1990. 
Sutton, M.A., Wolters, W.J., Peters, W.H., Ranson, W.F. and McNeill, S.R.: Determination of Displacements Using an Improved Digital Correlation Method. Im. Vis. Comp., 1(3): 133-139, 1983. 